\title{
Arquitectura inclusiva y su utilización como instrumento socializador en educación
}

\author{
Inclusive architecture and its utilization as a socializing instrument \\ in education
}

Jorge Raedó y

Santiago Atrio

\section{Introducción}

Los autores del presente trabajo, organizamos el "Encuentro Internacional de Infancia + Arquitectura + Educación + Inclusión" para conocer profesionales y proyectos que abordan la interrelación de las cuatro palabras que aparecen en su título.

En nuestro contexto internacional hay muchos autores y colectivos que abordan la temática del espacio arquitectónico educativo (Krauel \& Broto, 2010), (Meuser, Natascha (ED.), 2014), (Casqueiro, 2014), pero nuestro trabajo no incide tanto en el elemento construido como sobre la forma en la que se define este espacio y su relación con la función educativa del mismo. Los profesionales que se reunirán en enero en el MNCARS de Madrid, centrarán su reflexión sobre el ambiente inclusivo de los espacios arquitectónicos y su función social, desde las cuatro perspectivas: Infancia + Arquitectura + Educación + Inclusión.

Unos inciden más en "Infancia", el núcleo en torno al que crecemos y nos estructura, el aire profundo que respiramos aun en la vejez.(Atrio \& Eslava, Arquitectura en las Primeras Etapas de la Educación, 2018), (Atrio S. , El Educador: Constructor del Razonamiento. Arquitectura y Educación en Infantil y Primaria, 2002).

Otros enfatizan la vertiente "Arquitectura", el espacio que nos cobija y da forma, el lugar que construimos con nuestro deseo y pericia técnica. El niño comprende con el cuerpo y mente el ambiente que lo rodea si profundiza en las herramientas para leer el mundo y participar en su transformación. (Brosterman, 2013), (Wright, 1998).

Los hay que anclan su devenir profesional en la "Educación", trazando rutas pedagógicas que orientan el aprendizaje del niño a través de proyectos compartidos, consensuados y retadores que ordenan el espacio donde acontecen. (Tonucci, 1996), (Vecchi, 2013).

Centrados en la "Inclusión", todos escriben el poema donde cabe la infancia y juventud completa, sin excepción. No hay palabras equivocadas o prescindibles, todos los niños y niñas son necesarios en la sociedad que les dio a luz. 
Queremos aprender del trabajo de los otros, y del propio hecho de encontrarnos. Profesionales de primera talla mundial explican sus proyectos, los visibilizan ante la sociedad, acercan su antorcha de luz a la oscuridad pegadiza que no quiere irse. Todas las luces juntas son el amanecer.

\section{Psicología ambiental y ambientes educativos}

$\mathbf{E} \mid$ hecho axiomático de que la conducta acontece siempre y necesariamente en un entorno ambiental ha sido explicado de muy diversas formas en la historia del pensamiento. La filosofía, la historia de las religiones, la sociología, entre otros saberes, han venido señalando, desde antiguo, cómo ciertas cualidades del ambiente (su naturaleza sagrada o profana, clima, densidad de población, etc.) actúan como determinantes e incluso instigadores de una gran variedad de comportamientos Florencio jiménez Burillo,

(Jiménez Burillo, F. y Aragonés, J.I., (Eds.), 1986, pág. 21)

No somos los primeros en acercarnos a este área de trabajo, de conocimiento e investigación, que intenta analizar la influencia del espacio en el individuo y/o los colectivos humanos. En 1975 se publicaba el trabajo de David Canter y Peter Stringer con la colaboración de lan Griffiths, Peter Boyce, David Walters y Cheryl Kenny, por Surrey University Press. Su versión española la hizo en 1978 el Instituto de Estudios de Administración Local en Madrid, titulando su trabajo "Interacción Ambiental: aproximaciones psicológicas a nuestros entornos físicos." El trabajo recoge múltiples estudios de la década precedente que inciden sobre la relevancia del ambiente en la conducta humana desde múltiples enfoques académicos.

La psicología ambiental y la psicología social han venido desarrollando importantes trabajos desde la primera mitad del siglo XX. Se reconoce al científico social Roger Garlock Barker (1903-1990) como el fundador de la psicología ambiental y una figura destacada en dicho campo durante décadas por su desarrollo del concepto de configuración de comportamiento. Desde entonces las Áreas de mayor significación en la Psicología Ambiental (Jiménez Burillo, F. y Aragonés, J. I., (Eds.), 1986, págs. 24-26) serían:

1. Definición, modelos teóricos y técnicas de investigación en psicología ambiental.

2. Representación cognitiva del ambiente.

3. Evaluación del ambiente.

4. Rasgos de personalidad y medio ambiente.

5. Actitudes hacia el medio ambiente.

6. Percepción ambiental.

7. Influencias del medio ambiente urbano.

8. Influencias del medio ambiente físico natural.

9. Influencias del medio ambiente construido.

10. Conducta espacial humana. Intimidad y territorialidad.

11. Conducta ecológica responsable.

12. Hacinamiento y conducta.

13. Estrés y medio ambiente.

14. Tomas de decisión ambiental.

15. Psicología ecológica y análisis de escenarios.

16. Respuesta al paisaje. 


\section{taphiya $\mathbf{4 6}$}

Nuestro análisis académico se hace desde esas premisas y otras como las de los geógrafos urbanos, pero acercándonos al uso del espacio como instrumento educativo desde un punto de vista didáctico.

Coincidimos con estas disciplinas en (Jiménez Burillo, F. y Aragonés, J. I., (Eds.), 1986, págs. 24-26):

- una naturaleza interdisciplinar, confluyendo la Ecología humana, la Sociología, la Arquitectura y la Geografía, entre otras. En este sentido Wohlwill, citado por (liménez Burillo, F. y Aragonés, J.I., (Eds.), 1986) decía en 1984 que jel ambiente no está en la cabeza! Esta frase nos es especialmente significativa en el contexto del ambiente educativo inclusivo que deseamos analizar.

- En la carencia de una teoría comprehensiva.

- En un eclecticismo metodológico.

- En la más que estrecha unión entre teoría y práctica puesto que postulamos que lo que se hace se aprende mejor que si se lee o se observa.

- En la relevancia del control percibido sobre el medio como factor de bienestar humano.

- En el estudio de las variables físicas, como ruido, temperatura, etc.

- Junto con la Psicología Ambiental coincidimos en la falta de institucionalización y práctica profesional definida, cuestión que estuvimos analizando con la propuesta de un título propio de máster que ahora estamos revisando.

Pero no son las únicas disciplinas que se han aproximado al espacio (Bordes, 2012), (Romañá Blay, 2004), (Neufert, 2013 (16 edición, $5^{a}$ tirada)), (Atrio, Ruíz, \& Gómez Moñivas, Arquitectura en la formación de formadores: del tangram a los mosaicos nazaríes. Firmitas, utilitas, y 'venustas, 2015). Es relevante el trabajo del arquitecto Josep Muntañola Thornberg, que hace una relación de la arquitectura con la psicología, pasando por Aristóteles, Hipódamo de Mileto, Vitrubio, Alberti, Claude Perrault, hasta llegar a Walter Gropius y la Bauhaus.

El llamado movimiento moderno en la arquitectura de los años veinte no es, pues, a pesar de su contenido revolucionario, una excepción. Los escritos de sus arquitectos pretenden una y otra vez usar a su favor los últimos adelantos de las ciencias exactas y de las sociales, y en particular, las últimas ideas de la psicología. Así encontramos al psicoanálisis en los movimientos expresionistas de estos mismos años y la psicología de la Gestalt en los movimientos más racionales, e, incluso, un inicio de la visión ecopsicológica en las arquitecturas organicistas del norte de Europa defensoras, ya en los años veinte, de un proceso industrial respetuoso con la naturaleza y con las características climatológicas, culturales y ecológicas de cada país.

Muntañola en (Jiménez Burillo, F. y Aragonés, J.I., (Eds.), 1986, págs. 35-36)

Dentro de un colectivo donde abundan los arquitectos es fácil identificarse con los caminos recorridos por Muntañola en los que nos muestra cómo "la psicología ha ayudado en muchos momentos a la arquitectura a autodefinirse, a conocer las características de cada momento cultural y a prever las consecuencias del diseño."Muntañola en (liménez Burillo, F. y Aragonés, J. I., (Eds.), 1986, pág. 36). Del mismo modo nos advierte que los proyectos arquitectónicos y urbanísticos:

Han constituido un objeto de estudio difícil, pero sugestivo, para el psicólogo, que fácilmente demuestra que esos «objetos sociales» (ciudades o edificios) representan una cultura y una manera de ser y de vivir, abriendo nuevos caminos al conocimiento psicológico tradicional. La psicología ambiental ha sabido aprovechar esta compleja dialéctica.

Muntañola en (Jiménez Burillo, F. y Aragonés, J.I., (Eds.), 1986, pág. 36) 
Educadores, pedagogos, geógrafos, sociólogos, maestros, psicólogos, historiadores, antropólogos y muchos otros profesionales podemos reflexionar sobre esos «objetos sociales» para abrir nuevos caminos a la ciencia tradicional.

Como arquitecto, tengo aquí un consejo que dar a los psicólogos ambientales. Al evaluar un edificio hay que evaluarlo en relación a la situación social y a la compleja interacción entre forma y ritual que es la arquitectura. De poco sirve buscar variables absolutas. Muy recomendables los estudios psicofisiológicos de R. Küller en Suecia, en los que se ve con gran claridad la compleja solidaridad entre la reacción emotiva psicofisiológica y la situación social. Un mismo edificio, por ejemplo, de Le Corbusier, con una determinada densidad de personas en su interior, tiene efectos diferentes en los usuarios de acuerdo con su origen e identidad cultural. Este efecto cambia según cambian las condiciones de uso (tipo de actividad, densidad, variación de usos, tiempo, etc.), también de acuerdo con ciertas circunstancias sociales. Si comparamos los resultados con diferentes edificios de diferentes características arquitecturales, pero con la misma gente en su interior, tenemos unos resultados psicofisiológicos muy sugestivos, con leyes de covarianza invertidas y contrapuestas según el origen cultural.

Muntañola en (Jiménez Burillo, F. y Aragonés, J.I., (Eds.), 1986, pág. 40)

Esperemos que estas reflexiones nos acerquen a nuevas interacciones entre forma y ritual sin olvidarnos de las personas que los habitan y manteniendo la duda eterna de los diversos resultados sugestivos del trabajo que desarrollamos y proponemos en los espacios.

Los invitados al Encuentro son:

\section{"Proyectos de inclusión en el Museo Reina Sofía" de Elena Aparicio (MNCARS, Madrid)}

Si hay un museo en el Estado español que haya construido una narración lógica de su simbología reciente a partir de las obras de arte heredadas, es el Museo Reina Sofía. Podría contar otros cuentos, también lógicos, mas el trabajo hecho hasta ahora es emocionante. Es útil tener un cuento con el que estar de acuerdo o no.

EI MNCARS ejerce de plaza pública del estudio y difusión del arte del siglo XX y XXI de España, América Latina y mundo occidental en general. Plaza que quiere estar abierta a todos en su contenido y continente, dos aspectos siempre susceptibles de mejora para los que buscan la excelencia.

Los programas de inclusión del Museo Reina Sofía para infancia, jóvenes y adultos abordan la diversidad psíquica, física, cultural... Elena Aparicio nos contará ejemplos de algunos de ellos. El carácter referencial del MNCARS los convierte en más singulares si cabe.

\section{"Proyectos de inclusión en el MAXXI" de Marta Morelli (MAXXI - Museo Nazionale delle Arti del XXI Secolo, Roma)}

El MAXXI - Museo Nacional de las Artes del Siglo XX tiene la educación como uno de sus ejes básicos. El departamento de educación funciona desde los primeros días del museo. La arquitectura contemporánea es uno de los grandes temas abordados por la institución.

El objetivo principal del departamento de educación es dar herramientas a los niños y jóvenes para que lean el mundo construido que los rodea, lo analicen, interpreten, interpelen. Un segundo objetivo, después de que el niño alcance el

\section{|44}




\section{tarbiya $\mathbf{4 6}$}

primero, son los procesos de participación para la transformación de ese mundo, su mundo.

Esa labor transformadora del hoy sólo es posible con la inclusión de todos, sin distinción alguna. Por eso el MAXXI trabaja con los colegios del barrio Flaminio, donde está la sede del MAXXI, Ilenos de alumnos de lejanas procedencias: Europa, norte de África, Asia, América Latina... y con colegios de otros barrios con la misma o mayor diversidad de alumnado.

La especie humana necesita variedad para mezclarse, mejorar y sobrevivir en el entorno natural que la acoge y amenaza a la vez. La obra humana manifiesta esa necesidad de mezcla, protección e inclusión. La buena arquitectura tiene esas componentes. El departamento de educación del MAXXI, dirigido por Marta Morelli, construye el marco espaciotemporal pedagógico para que la infancia y juventud aprendan qué es y cómo nace la buena arquitectura.

\section{“Proyecto diseño participativo nuevo hospital infantil” de Hanna Kapanen (Design Museum, Helsinki)}

El Museo del Diseño en Helsinki ordena y visibiliza los principales diseños industriales de Finlandia: mobiliario del hogar, mobiliario urbano, vestuario, máquinas... La identidad de un pueblo manifiesta en las formas de los objetos cotidianos. Formas nacidas como respuesta sencilla a las necesidades diarias, inspiradas por la naturaleza circundante. El gesto básico y suficiente como signo de distinción.

Finlandia es una sociedad cohesionada y homogénea que comprende la fuerza de la unidad. Por ello su educación se centra en la integración de todos ciudadanos en el conjunto social. Los colegios son las puertas de entrada del niño en la sociedad. Si un niño o joven queda fuera de la dinámica del conjunto, será una nota adulta sin sentido en la composición coral.

Todas las actividades educativas del país quieren incluir a todos los pequeños. También las del Museo del Diseño. Por ejemplo, el proyecto capitaneado por Hanna Kapanen, del departamento de educación, donde un grupo de niños diseñaron elementos para un nuevo hospital infantil en Helsinki.

Hanna les propuso ponerse en la piel de dos personajes, niño y niña con problemas de salud, para entender sus necesidades y cómo el diseño de los objetos por ellos necesitados podrían hacerles la vida mejor, incluso ayudarles a curarse. Aśí, los alumnos diseñaron vestuario para los niños pacientes, enfermeros y médicos. Diseñaron mobiliario, objetos y ambientes para las habitaciones y otros espacios del nuevo hospital infantil, que se inauguró hace pocos meses.

\section{"Pedagogía Hospitalaria" de Uriel Espitia (Fundación Unimonserrate. Bogotá, Colombia)}

La enfermedad es compañera inseparable de algunos niños y jóvenes en todo el mundo. Son enfermedades crónicas que los ata a espacios hospitalarios o domésticos lejos de la riqueza social de una vida infantil "normal", sin el empuje y vigor que exige el descubrimiento del mundo.

La Pedagogía Hospitalaria quiere ser parte de ese viento que aporta motivación a los pequeños pacientes para superar su dolencia y crecer como personas. Eso es la educación, una pista de despegue hacia las estrellas inexploradas, el descubrimiento sin fin del Universo a través de las artes y técnicas heredadas. El principio de todo es la curiosidad, el hambre por jugar y conocer.

Hay dos caminos en la Pedagogía Hospitalaria: con voluntad "escolarizante" para que el niño obtenga la certificación académica de su país, y sin voluntad "escolarizante" enfocándose en la estimulación intelectual y emocional del pequeño para que el aprender sea medicina de recuperación. 
El Profesor Uriel Espitia sigue el segundo camino y ayuda a los pequeños pacientes a construir espacios "interiores" donde aferrar e impulsarse para crecer. Transformar los espacios físicos de los hospitales para cumplir objetivos pedagógicos es un deseo raramente saciado. La escasez económica o ética de la sociedad también es una enfermad crónica.

\section{"Empower Parents: familias, museos, autismo" de Laura Donis (Madrid).}

Usted percibe el mundo de una manera, ella de otra manera y yo de otra diferente. Según lo percibimos y lo procesamos en nuestros cerebros (y corazones) actuamos, nos movemos, deseamos en consecuencia.

Los llamados niños y niñas autistas perciben y procesan el mundo de un modo diverso al de la "mayoría". Por eso los \|lamamos especiales, distintos... porque su percepción, y por lo tanto su actuación es distinta al de la "mayoría". Esa diversidad hace que no encajen con las dinámicas espacio-temporales y formales construidas para el beneficio de la "mayoría".

Empower Parents es un grupo de familias con hijos o hijas diagnosticados con espectro autista. Trabajan junto a especialistas para que el mundo se adapte a las necesidades de sus hijos, y éstos al mundo impuesto. Llevan años visitando las exposiciones de arquitectura de la Fundación ICO donde retan a pequeños y adultos a congeniar, comunicar y entenderse con la palabra, el movimiento y la gráfica. Es cuestión de llegar a acuerdos donde todos están cómodos.

Laura Donis es la profesional que coordina el grupo Empower Parents, marcando objetivos a largo plazo y dirigiendo las etapas del medio y corto plazo. Una labor tejedora de voluntades y esperanzas de familias que modifica los comportamientos de todos nosotros en favor de la ciudad más suave.

\section{"Proyectos de inclusión en Casa da Musica" de Jorge Prendas (Casa da Musica, Porto)}

El departamento de educación fue el primero en abrir en la Casa da Musica, institución para todos los ciudadanos y todas sus músicas. La calidad la labor educativa, programación artística y singularidad del edificio sede, diseñado por Rem Koolhaas, lo situó rápido en el imaginario local e internacional. La música como arte que aúna y teje comunidad, como expresión natural de los humanos, forma de autoconocimiento personal y colectiva.

Jorge Prendas, director del departamento de educación desde los orígenes de Casa da Musica, y su equipo orienta su labor con perspectiva holística, entendiendo la música como manifestación de todo el ser en los espacios que habita, espacios que a su vez se modifican con la melodía de tales cuerpos.

Casa da Musica ofrece actividades a todos los grupos ciudadano: niños, jóvenes, adultos de todas las edades e intereses, ciudadanos de todas las diversidades. Algunos de esos proyectos son dirigidos a grupos que etiquetamos como diversidades específicas. Todas las melodías humanas caben en Casa da Musica.

\section{"A different light", film documental de Laurence Thrush (Los Ángeles)}

El Colegio Onerva en la ciudad de Jyäskylä, Finlandia, recibe a los niños y jóvenes con diversidad visual o auditiva más complicadas de todo el país. Es un colegio, residencia y centro de recursos. Acoge a unos 150 alumnos. Además atiende a alumnos con diversidad auditiva o visual y su profesorado de toda Finlandia. Los profesionales del Colegio Onerva son la "última frontera" a quien acudir cuando todo lo demás no ha funcionado. 
El colegio empezó el proceso de diseño del nuevo edificio hacia 2012 y lo inauguró en 2016. Es un edificio diseñado para necesidades concretas de las diversidades citadas, y para la infancia y juventud. Tengamos en cuenta que las necesidades arquitectónicas de los sujetos con diversidad auditiva son bastante distintas a los de diversidad visual. Integrar las dos fue el gran reto.

En definitiva, es un colegio normal, con niños que quieren aprender, jugar, crecer entre risas y aventura. Así lo vio el cineasta Laurence Thrush, quien grabó durante cuatro años decenas de horas de la vida cotidiana de los alumnos, profesores, especialistas. Cuatro años donde vemos el proceso de diseño y construcción del nuevo edificio. El resultado es el documental "Una luz diferente" que tiene su estreno mundial en este Encuentro.

Pequeños y adolescentes estudiando, practicando deporte, ensayando una ópera, haciendo excursiones aquí y allá, aprendiendo a moverse en el mundo, inventando siempre lo que está un poco más allá. Un colegio son sus alumnos y profesores, un proyecto pedagógico, una construcción de comunidad. Eso es la mano. Una mano concreta. El edificio será el guante que se adapte a esa mano.

\section{"Saludo del Colegio Onerva", vídeo de los alumnos y profesores (Jyäskylä, Finlandia)}

Los alumnos y profesores del Colegio Onerva que aparecen el documental "Una luz diferente" han grabado un vídeo de salutación para todos los asistentes al Encuentro. Vemos a las mismas personas años más tarde, más mayores, con otros objetivos y rutas. Una misma comunidad.

\section{"Neuroarquitectura e infancia" de Ana Mombiedro (Mallorca)}

En los últimos diez años se ha investigado el cerebro con intensidad. Cómo funciona, cómo evoluciona, cómo percibe y construye en mundo que nos aloja. Qué es la percepción visual, cómo nos orientamos, cómo construimos mapas mentales. Cómo se relaciona con el espacio que nos circunda, y por ende, cómo lo afecta. Todo ello tiene un desarrollo desde la gestación hasta la vejez. Cómo crece el cerebro del niño, cómo se relaciona con su cuerpo, cómo "inventa" del espacio que lo contiene.

¿Todos esos conocimientos sobre el cerebro nos ayudan a diseñar una mejor arquitectura, mejores hábitats, mejores escuelas? La arquitecta Ana Mombiedro ha volcado su investigación en la relación entre el cerebro y la arquitectura. A partir del mejor conocimiento científico de la "máquina de pensar" se diseña un entorno construido mejor adaptado a las necesidades de cada edad.

Si el arte -y la arquitectura es un arte- es un canal de conocimiento, si las obras de arte son objetos concretos -como los edificios, la ciudad y el paisaje- ("productos" los Ilaman algunos con tonillo despectivo) que encienden nuestra consciencia, nos hacen sentir el devenir que somos y nos nombra... ¿Cuál es la relación entre el cerebro y el arte?

Palabras del verso que no sale, melodía desviada aún, trazo falso antes de que nazca el verdadero... La voluntad busca con hambre algo que no existe sino en el horizonte interior. ¿Cuál es la relación entre el cerebro y la arquitectura? Salga por la puerta y busque.

\section{"Arquitectura escolar para la inclusión" de Ángel B. Comeras(Universidad San Jorge, Zaragoza)}

¿Qué es un colegio? Un lugar para aprender, dicen. Y hoy día, dicen, un lugar para aprender a aprender. Mañana tal vez digan que es un lugar para aprender a aprender a aprender. Mientras, los muros, suelo, techo, puertas, ventanas, pasillos, aulas, salas, escaleras, balcones, terrazas, porches, patios, jardines, huertos... del colegio son compañeros 
fieles de los alumnos y profesores durante sus largos años dentro de ellas. Estén o no bien diseñadas, construidas, mantenidas... apoyen bien o no el rumbo pedagógico marcado por los maestros, sea o no un buen refugio ante las inclemencias del tiempo... la infraestructura escolar está ahí con toda su gravedad. Mejor será entonces hacerlas bien.

Como barcos cruzando océanos de sorpresas, los colegios llevan a los alumnos y sus profesores por aventuras insospechadas donde la luz del estudio difumina los monstruos de la ignorancia. Tripulaciones donde todos entienden su lugar y cometido esencial en el grupo, así funciona un buen colegio. Según donde queramos ir, el barco será de una manera u otra; y la tripulación gobernará de un modo u otro.

Los colegios tienen que diseñarse a partir de un proyecto pedagógico claro, consensuado y querido. 0 de otro modo sólo serán cajas de zapatos donde cabe cualquier cosa y apenas flotan en el inmenso oleaje del día a día. Los arquitectos, profesorado, especialistas en espacios y materiales educativos, padres y alumnos tienen que trabajar juntos en el diseño de cada colegio que se adaptarán a contextos concretos.

Hay centros educativos especiales que acogen a alumnos que no pueden estar en otros centros. Todo se probó con el niño y no funcionó. Es mejor que existan centros especiales a modo de última frontera, el último faro de conocimiento y atención profesional hacia los niños más especiales. Más allá no haya nada, ni oscuridad. El arquitecto Ángel B. Comeras, especialista en arquitectura para la diversidad psíquica, ha estudiado algunos de estos centros educativos especiales y nos los contará.

\section{"El Juego de Alegría" de Sandra Montero (Zaragoza)}

La arquitecta y fotógrafa Sandra Montero tiene un hijo y dos hijas, una de ellas, Alegría, con hemiparesia derecha congénita. Desde que la diagnosticaron, Sandra documenta la evolución de su hija con fotos y textos. Una observación atenta a través del ojo de la cámara, a través del arte. Sus fotos hablan de una familia feliz que disfruta aprendiendo de todo lo que le rodea, en especial de la naturaleza del Pirineo aragonés. Caminan, ríen, pintan, corren y parecen bailar. Nos enseñan a todos una manera de vivir.

El cuerpo de la niña tiene dificultades en el movimiento, le cuesta caminar y explorar el mundo atractivo que tanto le Ilama la atención. Su cuerpo busca estrategias para adaptarse y sumarse al juego de su hermano. Cuerpo y mente son uno. Sus manos manchadas embadurnan la hoja con rayas de colores, palpa la materia y crea formas nuevas. Palabras, sonidos, luces... todo cabe en el Juego que Alegría inventa para nombrar su mundo, que ya es el nuestro.

¿El universo está ahí y nosotros lo descubrimos poco a poco? ¿0 el universo lo inventamos con cada gesto, cada paso, cada mirada? ¿Qué evidencia nuestra existencia? ¿El dolor? ¿La alegría? Lágrimas, risas, colores. Trazos arriesgados que manchan la hoja.

\section{"La mochila pedagógica del MNAC" de Joan Vitòria (El Globus Vermell, Barcelona)}

Los museos encuentran hoy su razón en la interacción con el ciudadano. Si éstos no se apropian del significado que el museo se otorga, el museo carece de sentido, está vacío de valor. Por ello los buenos museos construyen su significado a partir del roce con las comunidades que lo acogen.

El Museo Nacional de Arte de Catalunya participa en la construcción de la identidad catalana desde la perspectiva más oficial. El centro ordena el arte visual catalán en orden cronológico. Pintura, dibujo y escultura sobre todo. Reunidas en un mismo espacio, ordenadas de una cierta manera, las obras de arte parecen contar una historia concreta. Si las reuniéramos de otra manera, si las ordenáramos de otro modo, nos contaría otra historia. La memoria es frágil.

\section{$\mid 48$}


Llevar esa historia nacional a la infancia y juventud es una de las tareas del MNAC. Los colegios son "público cautivo", sí. Los proyectos educativos con ese público obligado pueden ser una tortura para los chavales si no se hacen con imaginación y pericia. Bien hechos, el museo se convierte en un actor pedagógico principal de los "paisajes educativos" que en sí son los barrios y la ciudad.

Joan Vitòria y su equipo de El Globus Vermell ha diseñado la nueva mochila pedagógica del MNAC, destinada a toda la infancia y juventud, sea cual sea su diversidad. La inclusión es el objetivo. Todo un reto para su resolución formal. Abrámosla.

\section{"El patio inclusivo" de Carme Cols y Josep Fernández (El Safareig, Torroella del Llobregat)}

El proyecto pedagógico tendría que pautar el diseño de toda la infraestructura escolar, incluido el patio escolar. La zona de descanso y juego no es un añadido superfluo a la zona "seria" de las aulas. Bien diseñado y usado es un potenciador de socialización, descubrimientos, sorpresas, intercambios, aprendizaje.

Sombras de los árboles en el cálido verano, canturreo de las fuentes, las zanjas del huerto con las incipientes hojas de patatas, lugares del habla, del silencio, de mirar las nubes, los areneros para los más pequeños junto a rocas y troncos... Gran escalinata, graderío para ver todo y nada. Un patio es un cúmulo de propuestas espaciales, cada una de una tonalidad distinta, como lo son las personas.

Carme Cols y Josep Fernández, profesores de colegio y especialistas en transformación de patios escolares, nos muestran ejemplo exitosos de patios que potencian la inclusión de todos los alumnos y alumnas, sean cuales sean sus gustos y diversidades. El patio debería ser un mezclador de imaginaciones, el equilibrado cóctel de las diferencias.

Cuentan que las nubes se educan en un colegio muy lejano. Antes de verlas nosotros como tales nubes blancas, son de mil colores y formas, algunas tan raras que ni nos lo creeríamos. El patio de ese colegio tiene forma de viento que mezcla todas las nubecillas en una sola nubaza tormentosa. Allí aprenden las maneras de llover y tronar con las que luego regarán la Tierra.

\section{"Escenarios educativos para erradicar la exclusión, el machismo y la Igtbfobia" de Ricard Huerta (Universitat de València)}

Le educación se da de dos maneras. La exo-somática y la endo-somática. La exo-somática es la que provocamos cuando explicamos esto es así 0 asá, enseñamos técnicas y procesos... es lo decimos. La endo-somática es la educación que acontece con el ejemplo del profesor, con lo no dicho.

Por ejemplo, una ciudad decide hacer un gran proyecto educativo en todos sus colegios sobre "la igualdad de género". La ciudad genera excelente materiales educativos, buenos seminarios para el profesor sobre el uso de los materiales y métodos de enseñanza del tema... Si los profesores no practican con el ejemplo la "igualdad de género", el alumno no aprenderá nada sobre ello.

La inclusión pasa primero de todo por nosotros mismos. ¿Estamos haciendo todo lo posible para limar las aristas de nuestra intolerancia? ¿Por qué pensamos esto o aquello de esa persona diferente? Sólo si hacemos este ejercicio cada día y mejoramos como profesionales y equipos de trabajo transmitiremos a los alumnos actitudes correctas de convivencia colectiva y profesionales.

Ricard Huerta, gran experto en el aprendizaje del arte en museos y otros escenarios educativos, conoce el valor del ejemplo que ejerce el profesor. Salir al escenario de la enseñanza exige al profesor un vestuario adecuado para cada 


\section{tapbiya $\mathbf{4 6}$}

historia. Cada alumno es una historia, un tiempo compartido que no volverá. Escucharlas sin prejuicios, acompañarlas sin molestar.

\section{Conclusión}

$\mathbf{E} \mid$ aprendizaje se halla en las conversaciones y en las interacciones de la comunidad y no tanto en algo que posea el individuo.

(Lucas \& Claxton, 2013, pág. 114)

El libro "Nuevas inteligencias, nuevos aprendizajes. Inteligencia compuesta, expandible, práctica, intuitiva, distributiva, social, estratégica, ética" (Lucas \& Claxton, 2013) es un texto que aborda nuevos enfoques sobre la catalogación de las diversas inteligencias. Conforme profundizamos en el conocimiento humano descubrimos nuevas sinergias entre disciplinas que nos acercan a postulados diferentes. Este compendio de estudios sobre diversas inteligencias van más allá de las inteligencias múltiples de Howard Gardner mostrándonos nuevos enfoques sobre un ámbito de conocimiento conocido.

La inteligencia, según dichos autores es, entre otras categorías: "distributiva, social y ética". En la propuesta de "Encuentro Internacional de Infancia + Arquitectura + Educación + Inclusión", pretendemos que nuestro análisis también lo sea, distributivo, social y ético.

- La inteligencia es distributiva si manifiesta las posibilidades que para el desarrollo de la inteligencia tienen las herramientas comunicativas de baja y alta tecnología. Igual que Lucas y Claxton, coincidimos con la conveniencia de la manipulación material y el uso de diversos lenguajes para aproximarnos a un aprendizaje profundo dejando que las nuevas tecnologías sean herramientas con las que analizar lo observado. "Puedes estar rodeado por todo tipo de herramientas inteligentes, pero si no estás dispuesto a hacer uso de las mismas, será como si no existieran." (Lucas \& Claxton, 2013, pág. 96).

- La inteligencia es social pues como ya apuntó Vygotsky el conocimiento se construye socialmente tanto con la interacción entre iguales como con aquellos que saben más y sobre todo menos que nosotros. En este sentido resultan reveladoras las investigaciones que apuntan que para que el alumnado desarrolle una inteligencia dinámica, creativa y productiva, el trabajo en grupo es esencial. "Bastan diez minutillos de debate con los demás para mejorar nuestro rendimiento mental." (Lucas \& Claxton, 2013, pág. 108).

- Y la inteligencia es ética, pues para nuestra problematizada aldea global de nada sirve un alumnado disciplinado si su formación académica no está acompañada de una "aversión hacia el daño ajeno" y un sentido de la "justicia, la lealtad al grupo y el respeto a la autoridad" (Lucas \& Claxton, 2013, pág. 151) como valores morales y capacidades esencial de los individuos. 


\section{Bibliografía}

Alćñtara García, P. (1874). Estudios pedagógicos. Froebely los Jardines de la infancia. Madrid.

ATRIO, S. (2002). El Educador: Constructor del Razonamiento. Arquitectura y Educación en Infantil y Primaria. Revista Educación y Futuro digital, 50-56.

ATRIO, S. y RAEDó, J. (Coordinadores). (10 de junio de 2016). Arquitectura y Educación para la Justicia Social. RIEJS. Revista Internacional de Educación para la Justicia Social, 250. doi: https://doi.org/10.15366/riejs

Atrio, S. y ESLAVA, C. (2018). Arquitectura en las Primeras Etapas de la Educación. Educación y Futuro (39). (En Prensa).

Atrio, S.; Raedó, J. y NavarRo, V. (1 de Noviembre de 2016). Educación y Arquitectura: ayer, hoy, mañana. Crónica del III Encuentro Internacional de Educación. (F. Arroyo Llera, Ed.) Tarbiya, Revista de Investigación e Innovación Educativa (IUCE)(44), 131, 148. doi: http://dx.doi.org/10.15366/tarbiya2016.44

ATRIO, S.; RuÍZ, N. y GÓmez MoÑIIAS, S. (2015). Arquitectura en la formación de formadores: del tangram a los mosaicos nazaríes. Firmitas, utilitas, y'venustas. Bordón. Revista De Pedagogía (68(1)), 43-59.

doi: http://dx.doi.org/10.13042/Bordon.2016.68103

BORDES, J. (2012). Historia de los juguetes de construcción. Escuela de la arquitectura moderna. Madrid: Ediciones Cátedra (Grupo Anaya, S.A.).

BrosteRman, N. (13 de 06 de 2013). Inventing Kindergarten by Norman Brosterman book on Froebel Design Influence Kickstarter.

https://www.youtube.com/watch?v=Y_wZhEAlk3U

CasqueIRO, F. (2014). Canon de Centros Escolares del sig/o XX. Grupo de Investigación ARKRIT. Madrid: MAIREA LIBROS.

ComenIus, A. (02 de marzo de 2018). FPschool. (1 $1^{a}$ edición 1633-1638). Obtenido de Didáctica Magna: http://fpschool.es/doc/www.saviadigital.com.pdf

Conjuntos Empáticos. (enero de 2018). Ludantia. Obtenido de Web oficial de la I Bienal Internacional de Educación en arquitectura para la infancia y juventud:

http://ludantia.wixsite.com/bienal-internacional/convocatoria-proyecos-educativos

FröBeL, F. (1861). L'éducation de / 'homme. (B. d. Crombrugghe, Trad.) Bruselas: Typographie de veuve J. van Buggenhoudt.

JImÉnez BuRILLO, F. y Aragonés, J.I. (Eds.). (1986). Introducción a la Psicología Ambiental. Madrid: Alianza Editorial, S. A. $\left(1^{\mathrm{a}}\right.$ ed. $)$

Krauel, J. y Broto, C. (2010). Arquitectura para la Educación. Barcelona: Links/Structure.

LUCAS, B. y CLAXTON, G. (2013). Nuevas inteligencias, nuevos aprendizajes. Inteligencia compuesta, expandible, práctica, intuitiva, distributiva, social, estratégica, ética. Madrid: Narcea.

MEUSER, N. (ED.). (2014). School Buildings. Construction and Design Manual. Berlín: DOM PUBLISHERS.

Neufert, E. (2013). Arte de Proyectar en Arquitectura. Barcelona: Gustavo Gili. (16ª edición, 5 a tirada)

Romañá Blay, T. (2004). Arquitectura y educación: perspectivas y dimensiones. Revista Española de Pedagogía, 62 (MONOGRÁFICO CORRESPONDIENTE A LA EDICIÓN: N. $\left.{ }^{\circ} 228\right), 199-220$.

https://revistadepedagogia.org/monografico/arquitectura-y-educacion/ 
taphiyg $\mathbf{4 6}$

Tonuccl, F. (1996). La ciudad de los niños: un modo nuevo de pensar la ciudad. Madrid: Fund. Germán Sáanchez Ruipérez.

VECCH, V. (2013). Arte y creatividad en Reggio Emilia. Madrid: Ediciones Morata.

WRIGHT, F. L. (1998). Autobiografía: 1867-1944. Madrid: El Croquis. 


\section{Resumen.}

En la actualidad son muchos los colectivos que trabajan con el espacio como instrumento educativo (Conjuntos Empáticos, 2018). Son herederos, a veces sin saberlo, de pedagogos como Johann Amos Comenius (1592-1670), (Comenius, 2018, primera edición 1633-1638), Johann Heinrich Pestalozzi (1746-1827), Friedrich Fröbel (17821852)(Alcántara García, 1874), (Fröbel, 1861), Caroline Pratt (1867-1954)(Atrio \& Eslava, Arquitectura en las Primeras Etapas de la Educación, 2018). Y provienen de diferentes formaciones universitarias y su trabajo se desarrolla en todo el mundo (Atrio, S. y Raedó, J. (Coordinadores), 2016). Este colectivo continúa investigando sobre cómo se utiliza el espacio como instrumento educativo y cómo contribuir a consolidar un área de conocimiento interdisciplinar. El grupo viene desarrollando su trabajo con diferentes edades pero son los menores de 18 años, la que atienden especialmente. Con estos antecedentes (Atrio, Raedó, \& Navarro, Educación y Arquitectura: ayer, hoy, mañana. Crónica del III Encuentro Internacional de Educación, 2016), se ha creído necesaria la reflexión sobre el espacio, la educación, en relación con la inclusión educativa y por ello surge la iniciativa de desarrollar un "Encuentro Internacional de Infancia + Arquitectura + Educación + Inclusión" que se desarrollará los días 19 y 20 de enero de 2019 en el Museo Nacional Centro de Arte Reina Sofía de Madrid MNCARS. No se trata de hacer espacios educativos que atiendan las necesidades especiales de colectivos concretos, sino de hacer espacios inclusivos, pensados por y para todo tipo de públicos. El encuentro está organizado por la Escuela en Arquitectura Educativa de la UAM, adscrita tanto al Instituto Universitario de Ciencias de la Educación IUCE, como a la Facultad de Formación de Profesorado y Educación, ambos de la Universidad Autónoma de Madrid.

Palabras clave. Calidad de la educación, Centro de educación especial, Ambiente educativo, Enseñanza de la arquitectura, Espacio de juegos, Derechos de grupos especiales.

\section{Abstract.}

Nowadays there are many groups that work with space as an educational tool (Conjunto Empáticos, 2018). They are heirs, sometimes without knowing it, of pedagogues like Johann Amos Comenius (1592-1670), (Comenius, 2018, first edition 1633-1638), Johann Heinrich Pestalozzi (1746-1827), Friedrich Fröbel (1782-1852) (Alcántara García, 1874), (Fröbel, 1861), Caroline Pratt (1867-1954) (Atrio \& Eslava, Architecture in the First Stages of Education, 2018). And they come from different university backgrounds and their work is developed all over the world (Atrio, S. and Raedó, J. (Coordinators), 2016). This group investigates how space is used as an educational tool and how to contribute to and how to contribute to consolidate an interdisciplinary area of knowledge. The group has been developing their work with different ages, but they usually attend people younger than 18 years old. With this background (Atrio, Raedó, \& Navarro, Education and Architecture: yesterday, today, tomorrow, Chronicle of the III International Meeting of Education, 2016) it was necessary to think on space, education, in relation to educational inclusion and to develop the "International Encounter of Children + Architecture + Education + Inclusion" that will be held on January 19th and 20th, 2019 at the Reina Sofía National Art Museum of Madrid MNCARS. This is not about making educational spaces for specific groups with special needs, but about making inclusive spaces, designed by and for all types of audiences. The meeting is organized by the School of Educational Architecture of the UAM, attached to the University Institute of Education Sciences IUCE, and the Faculty of Teacher Training and Education, both of the Autonomous University of Madrid.

Key-words. Educational quality, Special schools, Educational environment, Architecture education, Playgrounds, Rights 
taphiya $\mathbf{4 6}$

of special groups.

Jorge Raedó

Artista especialista en Educación de Arte/Arquitectura para infancia y juventud

Santiago Atrio Cerezo

Departamento de Didácticas Específicas

Facultad de Formación de Profesorado y Educación

Universidad Autónoma de Madrid

IUCE. UAM

santiago.atrio@uam.es 